\title{
Dimensions of social media addiction among university students in Kuwait
}

\author{
Jamal J. Al-Menayes \\ Kuwait University, College of Arts, Department of Mass Communication, Kuwait City, Kuwait
}

Email address:

jamal@almenayes.com

To cite this article:

Jamal J. Al-Menayes. Dimensions of Social Media Addiction among University Students in Kuwait. Psychology and Behavioral Sciences. Vol. 4, No. 1, 2015, pp. 23-28. doi: 10.11648/j.pbs.20150401.14

\begin{abstract}
This study aimed to examine social media addiction in a sample of university students. Based on the Internet addiction scale developed by Young (1996) the researcher used cross-sectional survey methodology in which a questionnaire was distributed to 1327 undergraduate students with their consent. Factor analysis of the self-report data showed that social media addiction has three independent dimensions. These dimensions were positively related to the users experience with social media; time spent using social media and satisfaction with them. In addition, social media addiction was a negative predictor of academic performance as measured by a student's GPA. Future studies should consider the cultural values of users and examine the context of social media usage.
\end{abstract}

Keywords: Social Media, Addiction, Factor Analysis, Kuwait

\section{Introduction}

Internet addiction is not yet considered a disorder by the psychiatric literature as evidenced by its exclusion from the Diagnostic and Statistical Manual of Mental Disorder (DSM-V), published by the American Psychiatric Association. However, an alarming rate of people show what are seemingly symptoms of addiction to Cyberspace. Young people seem especially susceptible, with evidence underscoring students whose academic performance is compromised as they spend increasing amount of time online (Al-Menayes, 2014). Some also suffer health consequence resulting from lack of sleep brought about by the growing amount of time they spend on the Internet especially late at night.

Research into Internet addiction has grown dramatically since the mid 1990s, especially as more and more cases among college students have been detected by university healthcare professionals (Wallace, 2014). The terminology, to describe this phenomenon, varies widely in the literature. In addition to 'Internet addiction', terms such as 'Internet dependency', 'compulsive Internet use', 'problematic Internet use', 'dysfunctional Internet use', and 'pathological Internet use' have been used to describe what is essentially the same behavior (Kuss and Griffiths, 2012). For this article, I will use 'Internet addiction' mainly due to its wide usage in the research.
Research in different countries has produced varying results of the prevalence of Internet addiction. A study in the UK, for example, found Internet addiction to be prevalent among $18 \%$ of young people (Neimz, Griffith and Banyard, 2006). A study in Italy found that rate to be only $0.8 \%$ (Poli and Agrimi, 2012). In addition, a large sample survey in China puts the rate at $12 \%$ among male and $5 \%$ among female students (Lau, 2011). Internet addiction is not just restricted to college campuses; it also extends to high school as well as middle school students. A longitudinal survey conducted in Hong Kong reported prevalence rate of Internet addiction as high as 26.7\% among high school students (Yu and Shek, 2013).

When it comes to the amount of time spent online, studies show that individuals, who regard themselves as Internet addicts, indicated that it varies greatly from 8.5 hours per week to 21.2 hours per week (Yang and Tung, 2007). Other studies found that the higher the amount of time spent online, the greater the extent of the symptoms of Internet addiction (Leung, 2004; Widyanto and McMurran, 2004).

In relation to users' psychological profile, studies have revealed a correlation between depression, locus of control, loneliness, social anxiety, self-esteem and Internet addiction (Selfhout et al., 2009; Sun et al., 2005). Whang et al. (2003) found that Internet addicts had a higher degree of loneliness 
and depression compared to non-addicts. Other findings revealed that computer self-efficacy was a significant correlate of problematic Internet use. Internet addiction was also associated with poor mental health and low self-esteem in adolescents (Yen et al.., 2009).

\section{Social Media Addiction}

While none of the previous studies addressed the use of mobile social media per se, it is safe to say the results of computer-based Internet addiction also apply to mobile Internet since they both use the same medium essentially. The introduction of anytime anywhere $\mathrm{Wi}-\mathrm{Fi}$ in mobile phones and the prevalence of free social media apps made them indistinguishable from personal computers when it came to Internet addiction. In addition, as their name indicates, mobile phones are portable providing easy access to the Internet regardless of time and place. This makes them the ideal medium for Internet addicts.

Mobile social media offer a large number of experiences from a psychological viewpoint, each with powerful features that can lead to problem behavior. For example, the extrovert might spend much time on Facebook, compulsively checking their profile to see the number of 'likes' their latest post received. For others, with a narcissistic inclination, Instagram may prove to be an addictive medium for them to display themselves to others with 'selfies.' Social anxiety can also fuel social media addiction. The fear of missing out (FOMO) can be the main reason for frequent social media use regardless of time of day at the expense of other activities (Przybylski et al.., 2013).

'Mobile phone addiction' is sometimes used to distinguish the concept of Internet addiction. Most of the traditional studies of online addiction do not address problematic mobile phone use. Mobile phones today offer access to almost all Internet applications along with voice and video calls, text messaging, video recording and a myriad of engaging apps designed especially for small screens. Additionally, their results can also be shown on any screen. In addition, they have the added dimension of being always available, unlike a desktop or even a laptop.

The mobile phone can be used while walking, riding on public transportation and even while driving. These 'micro time slots' in which people can engage in a multitude of online activities were not previously available. This can lead to obsessive mobile phone usage and can interfere with face-to-face interaction and harm academic performance (Almenayes, 2014).

Research on problematic mobile media usage is limited but has attracted increasing attention recently. A study of Taiwanese female university students, for example, found that students, who scored high on a test of mobile phone addiction, showed more extraversion and anxiety, and somewhat lower self-esteem (Fu-Yuan and Chiu, 2012). Women seem to be more vulnerable to mobile phone addiction than men.

Another feature of mobile phones, that may prove to be of particular importance to addictive behavior, is 'texting' either directly or through social media such as Twitter and similar applications. Recent surveys indicate that young people are starting to discard Facebook in favor of Twitter, particularly as their parents create accounts and ask to be 'friended' (Madden et al.., 2013). These types of applications are growing and allowing more and more features such as Vine, which allows users to create six-second videos to share with followers. The overarching feature of these applications is their 'stickiness', the propensity to have users utilize the app frequently. Stickiness is a result of their business models that rely on the growing heap of data on user behavior to share with advertisers for targeted marketing.

\section{Research Questions}

Building on previous research, in which investigators measured Internet addiction in a variety of domains; my first research question addresses the dimensions of social media addiction. The issue here is whether addiction is manifested in a single factor or a multitude of factors. The second research question takes the investigation a step further by addressing the correlates of social media addiction in terms of patterns of usage and satisfaction with the medium. The third question addresses the effects of social media addiction on academic performance. These questions are as follows:

RQ1: What are the underlying factors behind social media addiction?

RQ2: What are the correlates of social media addiction?

RQ3: Does social media addiction affect academic performance?

\section{Method}

\subsection{Sample and Procedures}

A self-administered survey questionnaire was used for this study. Because young people constitute the core users of social media, the data were collected from a sample of purposively selected college students. College students enrolled in coursework in mass communication at a large state university in Kuwait were asked to participate in this study. The questionnaires were distributed over a period of three months starting in March 2014. The total sample size was 1327. Arabic was the language used in the questionnaire.

Students were assured of anonymity and confidentiality, and participation was voluntary. The age of the participants ranged from 18 to 31 with $96 \%$ ranging between 18 to 25 years of age. The mean age of the participants in the study was 21.87 years. The participants were $395(29.8 \%)$ male and 931 $(70.2 \%)$ female. This gender distribution reflects the enrollment profile of the university student body that is $70 \%$ female. Finally, since this is a state university, the overwhelming majority of students are Kuwaiti nationals by law, so there was no need to record the nationality. The self-administered questionnaires were distributed during regularly scheduled class sessions. The instrument consisted of both Likert scale questions used to measure the individual's perceptions, attitudes, and behaviors, as well as demographic 
questions and questions about media, use patterns.

\subsection{Measurement}

\subsubsection{Social Media Addiction}

A Likert scale consisting of fourteen items was used to measure social media addiction. This scale was based on Young's (1996) measurement of Internet addiction, and the response set ranged from 'strongly disagree' (1) to 'strongly agree' (5). Table 1 shows the English translation of the items that were worded in Arabic.

Table 1. Wording of the Social Media Addiction items included in the factor analysis (original in Arabic).

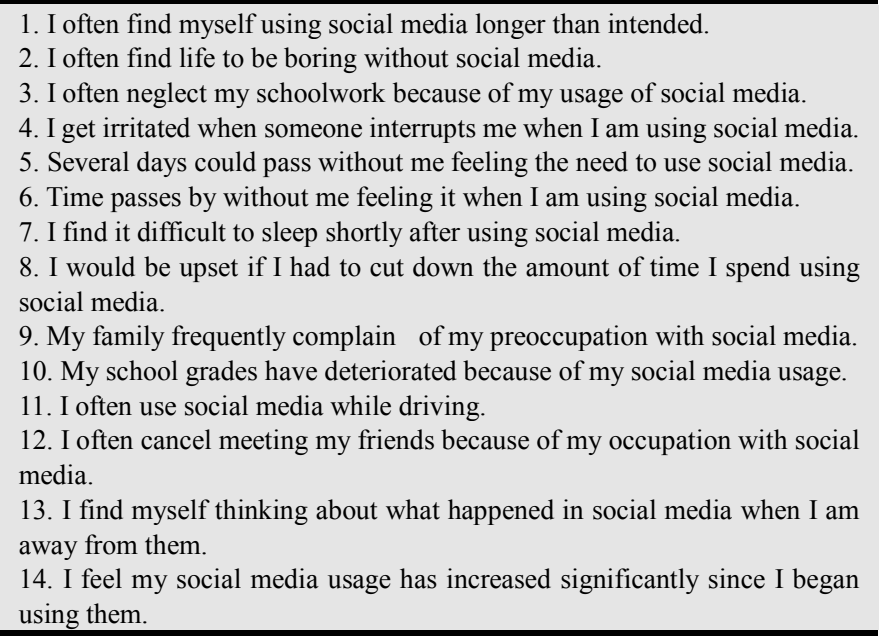

\subsubsection{Experience with Social Media}

Respondents were asked a single question about the time they first started using social media. The response set ranged from (1) less than a year to (8) more than six years.

\subsubsection{Hours Spent Using Social Media per Day}

Respondents were asked a single question about the total number of hours spent using social media daily on an eight-point scale: (1) less than two hours, (2) from two to 4 hours, (3) from 4 to 6 hours, (4) from 6 to eight hours, (5) from eight to 10 hours, (6) from 10 to 12 hours, (7) from 12 to 14 hours, (8) more than 14 hours.

\subsubsection{Satisfaction with Social Media}

Similar to Palmgreen and Rayburn (1985), the study used a single-item to measure satisfaction with social media use. Respondents were asked to indicate: "Overall, how satisfied are you with social media in what it does in providing you with the things you are seeking?" Response options ranged from extremely satisfied (5) to not at al satisfied (1). This measure had a mean of $4.00(S D=0.79)$.

\section{Results}

\subsection{Descriptive Statistics of Social Media Use Patterns}

Before I proceed to the data analysis, I will first present some key descriptive statistics of the sample relevant to the research questions. Table 2 contains answers to questions about whether or not respondents use social media at all, whether they use social media while driving and if they met anyone in person through social media. Ninty nine percent of the sample reported they used social media, 51\% said they used it while driving, and $58 \%$ mentioned they met someone in person he or she first ran into on social media.

Table 2. Descriptive summary of social media uses patterns.

\begin{tabular}{lll}
\hline Variable & Yes (\%) & No (\%) \\
\hline Do you use social media (SM)? & $1316(99)$ & $10(0.8)$ \\
Do you use SM while driving? & $648(51)$ & $676(49)$ \\
Have you met anyone in person through SM? & $771(58)$ & $555(42)$ \\
\hline
\end{tabular}

$n=1326$

Table 3 ranks the respondents favorite social media apps. Whatsapp is by far the favorite (50\%) followed by Instagram $(23 \%)$, and lastly Twitter (16\%).

Table 3. Popularity of social media applications.

\begin{tabular}{ll}
\hline Which social medium do you use the most? & $\boldsymbol{n}(\%)$ \\
\hline Whatsapp & $663(50)$ \\
Instagram & $302(23)$ \\
Twitter & $216(16)$ \\
Others & $111(8)$ \\
\hline
\end{tabular}

Valid $n=1293$

\subsection{Dimensions of Addiction to Social Media}

Fourteen Likert-scale items were used in the questionnaire to estimate the dimensions underlying our key variable "social media addiction." Exploratory factor analysis with varimax rotation was performed on these items to ascertain their underlying factors. As a result of the analysis, four items were discarded due to loadings under 0.5. All items were standardized to ensure they were on equal footing.

Table 4 shows the results of this analysis. As we can see, there are three factors representing the underlying dimensions of addiction to social media. Each factor represents a different grouping of addiction items. Factor 1 represents deterioration of school performance, driving, not meeting friends and thinking about social media when not using them. Factor 2 reflects social media overuse, neglecting schoolwork, feeling irritable and lack of sleep due to social media usage. Factor 3 has two items only, one dealing with boredom and the other with the need to use social media.

Table 4. Factor analysis of Social Media (SM) Addiction Scale with Varimax Rotation.

\begin{tabular}{llcc}
\hline Factors & Mean & SD & 1 \\
\hline Factor 1 & & & \\
1. Grades deteriorated because of SM. & 2.44 & 1.24 & 0.636 \\
2. I often use SM while driving. & 2.66 & 1.36 & 0.560 \\
3. I cancel meeting my friends because of SM. & 1.67 & 0.97 & 0.787 \\
4. I think about SM when I am away. & 3.03 & 1.24 & 0.582 \\
\hline
\end{tabular}




\begin{tabular}{|c|c|c|c|c|c|}
\hline Factors & Mean & SD & 1 & 2 & 3 \\
\hline \multicolumn{6}{|l|}{ Factor 2} \\
\hline 5. I find myself using SM longer than intended. & 4.20 & 0.95 & & 0.713 & \\
\hline 6. I neglect my schoolwork because of SM. & 3.25 & 1.22 & & 0.620 & \\
\hline 7. I get irritated when interrupted using SM. & 3.25 & 1.20 & & 0.509 & \\
\hline 8. It is difficult to sleep after using SM. & 2.89 & 1.28 & & 0.530 & \\
\hline \multicolumn{6}{|l|}{ Factor 3} \\
\hline 9. I find life boring without SM. & 3.98 & 1.09 & & & 0.622 \\
\hline 10. Days pass by without the need to use SM.* & 2.66 & 1.32 & & & -0.762 \\
\hline Eigenvalue & & & 2.70 & 2.66 & 1.87 \\
\hline$\%$ of the variance explained & & & 19.30 & 19.01 & 13.35 \\
\hline Cronbach's alpha & & & 0.70 & 0.63 & 0.94 \\
\hline
\end{tabular}

Notes: Loadings $<0.50$ were suppressed. * Item wording was reversed for reliability analysis.

\subsection{Correlates of Social Media Addiction}

To examine the relationship of social media addiction with usage patterns a correlation was performed. The variables included in the correlation are experience with social media, time spent using social media and satisfaction with social media. As Table 5 shows, there are strong correlations between all three variables. Experience with social media is positively correlated with factors 2 and 3, but is negatively correlated with factor 1 . Time spent using social media is positively correlated with all three addiction factors. Satisfaction with social media is also positively correlated with all three factors of addiction.

Table 5. Pearson correlations between SM addiction factors and social media variables.

\begin{tabular}{llll}
\hline Variables & Start & Time & Satisfaction \\
\hline Factor 1 & $-.075^{*}$ & $.170^{*}$ & $.077^{*}$ \\
Factor 2 & $.130^{*}$ & $.278^{*}$ & $.108^{*}$ \\
Factor 3 & $.158^{*}$ & $.299^{*}$ & $.239^{*}$ \\
\hline
\end{tabular}

$* p<.001$

\subsection{Social Media Addiction and Academic Performance}

Linear regression was performed to examine the effect of social media addiction on academic performance. The three addiction factors were entered separately as independent variables and a student's grade point average (GPA) was used as the dependent variable. Table 6 shows results of this analysis. Addiction factor 1 is a strong negative predictor of GPA. This result means the higher a student scores on this factor, the lower his or her GPA will be. A similar result can be seen in factor 2 with it being a strong negative predictor of GPA. However, the same is not true of factor 3 that has no significant statistical relationship between GPA.

Table 6. Regressing Social Media Addiction Factors on a Student's GPA

\begin{tabular}{lllll}
\hline $\mathbf{T}$ & $\boldsymbol{\beta}$ & SE B & B & Variable \\
\hline$-3.07 * *$ & -.091 & .016 & -.049 & Factor 1 \\
$-2.54 * *$ & -.076 & .016 & -.040 & Factor 2 \\
-.48 & -.015 & .016 & -.00 & Factor 3 \\
\hline
\end{tabular}

Note: For factor $1, \mathrm{R}=.091, \mathrm{R}^{2}=.008, \mathrm{~F}=9.46^{* *}, \mathrm{df}=1$. For factor $2, \mathrm{R}=.076$, $\mathrm{R}^{2}=.006, \mathrm{~F}=6.47^{* *}$,

$\mathrm{df}=1$. For factor $3, \mathrm{R}=.015, \mathrm{R}^{2}=.000, \mathrm{~F}=.240, \mathrm{df}=1 . * \mathrm{p} \quad .05, * * \mathrm{p} \quad .001$

\section{Conclusion}

This study sought to examine the dimensions of social media addiction and their correlates. Previous research has shown evidence of what can be classified as an addiction (Al-Menayes, 2014). To investigate the matter further, the current study used an established Internet addiction scale and adapted it to social media (Young, 1996). Based on factor analysis of responses from a sample of 1326 respondents, three addiction factors emerged. These dimensions were subsequently correlated with communication related variables to measure their impact. Results indicated that the amount of time spent using social media is positively correlated with all factors of social media addiction meaning the more time one spends using social media, the more likely they will exhibit symptoms of social media addiction. Satisfaction with social media was also positively correlated with social media addiction. This means that individuals reporting signs of addiction are more likely than others to be satisfied with the functions social media provide. Experience with social media was positively correlated with two of the three factors while negatively correlated with the third. This means that generally the more experience a person has with social media, the more likely he or she will be addicted to them.

To examine the effect social media addiction has on real life outcomes a student's GPA was regressed on all addiction factors. Factors 1 and 2 were strong negative predictors of GPA. This indicates that individuals showing signs of social media addiction will have a lower GPA than those who do not. Social media usage, in this context, comes at the expense of academic performance. This appears to be the result of time displacement meaning the time spent using social media displaces the time usually allocated to studying. As with all addictions, social media usage interferes with the normal functioning of daily life, in this context academic performance. A useful path for further research would take into account the cultural values and their impact on the relationship between addiction and its correlates. Future studies should also continue to examine other possible motivation factors through more qualitative methods such as focus groups and participant observation. This methodological approach known as "triangulation" will help increase the depth and complexity of the variables used in the future. It will also enable us to take into account any future trends given that we are dealing with a 
phenomenon populated largely by youth.

\section{Limitations}

There are a few limitations that might affect the generalizability of the findings. First, the cross-sectional data utilized for this study do not merit an assertion of any causal relationships between the independent and dependent variables. In addition, the sample that had female to male ratio of $2: 1$ could skew the results by showing more variance in the former compared to the latter. Perhaps a quota sample with equal numbers of males and females should have been used to ensure that we do not get gender differences because of the uneven distribution.

Second, the definition of some constructs might limit the scope of the study. The main study variables were based on self-reports. For example, the variable "time spent using social media" was measured by asking participants how much time they spend using social media on a typical day. Although this question measures usage time accurately, some doubt remains as to whether users are active all the time they are logged on to a specific application. Heavy and light users can be better analyzed in future studies by asking how many messages are sent or received each day.

Third, the definition of typical social media use provided a viable empirical description to examine the research questions, but it might not precisely reflect the complexity of an individual's use patterns. It is possible that each uses several social media functions (e.g. Chat, post pictures, audio or video) each day. Researchers would benefit from developing tools for capturing the complexity of social media and user patterns.

Finally, the fact that data collected for this study of social media use was limited to college students should be taken into consideration. Investigating only college students' social media usage might not wholly explain the electronic social networking behavior, in general. Future researchers are also strongly encouraged to attempt to replicate these results by analyzing users of different social media platforms (e.g. Twitter, Instagram....etc.) separately to account for the various features they offer.

\section{Acknowledgements}

This research was supported and funded by Kuwait University Research Grant No. AM02/2014.

\section{References}

[1] Al-Menayes, J. (2014). The relationship between mobile social media use and academic performance in university students, New Media and Mass Communication, vol. 25, pp. 23-29.

[2] Fu-Yuan Hong and Chiu, D. H. (2012). A model of the relationship between psychological characteristics, mobile phone addiction and use of mobile phones by Taiwanese university female students, Computers in Human Behavior, vol. 28 , pp. $2152-2159$.
[3] Kuss, D. and M. Griffiths (2012). Internet gaming addiction: a systematic review of empirical research. International Journal of Mental Health Addiction, vol. 10, pp. 278-296.

[4] Lau, C. H. (2011). Internet addiction among university students in China. Dissertation: The Chinese University of Hong Kong.

[5] Leung, L. (2004). Net-generation attributes and seductive properties of the Internet as predictors of online activities and Internet addiction, Cyberpsychology and Behavior, vol. 7, pp. 333-348.

[6] Madden M, Lenhart A, Cortesi S, Gasser U, Duggan M, Smith A, Beaton M (2013). Teens, social media, and privacy. Pew Research Center. http://www.pewinternet.org/ /media//Files/Reports/2013/PIP TeensSocialMediaand- Privacy.pdf.

[7] Niemz, K., Griffiths, M. and Banyard, M. (2006). Prevalence of pathological Internet use among university students and correlations with self-esteem, Cyberpsychology and Behavior, vol. 11, pp. $480-483$.

[8] Palmgreen, P., and J. D. Rayburn (1985). A comparison of gratification models of media satisfaction. Communication Monographs, 52, 334-346.

[9] Poli, R. and E. Agrimi (2012). Internet addiction disorder: prevalence in Italian student population, Nordic Journal of Psychiatry, vol. 66, pp. 55-59.

[10] Przybylski, A., Murayama, K., DeHaan, C. and V. Gladwell (2013). Motivational, emotional, and behavioral correlates of fear of missing out, Computers in Human Behavior, vol. 29, pp. 1841-1848.

[11] Selfhout, M. H., Barnje, S. J. Delsing, M., Bogt, T.F. and Meeus, W. H. J., (2009). Different types of Internet use, depression, and social anxiety: The role of perceived friendship quality, Journal of Adolescence, vol. 32, pp. 819-833.

[12] Sun, P., Unger, J. B., Palmer, P. H., Gallaher, P., Chou, C. P., \& Baezconde-Garbanati, L. (2005). Internet accessibility and usage among urban adolescents in southern California: implications for web-based health research. Cyberpsychology \& Behavior, vol. 8, 441-453.

[13] Yang, S. and Tung, C. (2007). Comparison of Internet addicts and non-addicts in Taiwanese high schools, Computers in Human Behavior, vol. 23, pp. 79-96.

[14] Yu, L. and D. Shek (2013). Internet addiction in Hong Kong adolescents: A three-year longitudinal study, Journal of Pediatric Adolescent Gynecology, vol. 26, pp. 10-17.

[15] Wallace, P. (2014). Internet addiction disorder and youth, EMBO Reports, vol. 15, no. 1, pp. 12-16.

[16] Whang, L. S., Lee, S., \& Chang, G. (2003). Internet over-user's psychological profiles: A behavior sampling analysis on Internet addiction. Cyberpsychology \& Behavior, 6, 143-150.

[17] Widyanto, L. and McMurran, M. (2004). The psychometric properties of the Internet addiction test. Cyberpsychology and Behavior, vol. 7, 443-450.

[18] Yen, C. F., Ko, C. H., Yen, J. Y., Chang, Y. P., \& Cheng, C. P. (2009). Multi-dimensional discriminative factors for Internet addiction among adolescents regarding gender and age. Psychiatry and Clinical Neuro- sciences, 63, 357-364. 
[19] Young, K. S. (1996). Internet addiction: The emergence of a new clinical disorder. Cyberpsychology and Behavior, vol. 1, 237-244. 